\title{
SIMILARITY OF CHANGES IN AVERAGE PRICES OF RESIDENTIAL PROPERTIES IN EUROPE IN 2010-2016
}

\author{
Iwona Dittmann, PhD \\ Faculty of Finance \\ University of Economics in Wroctaw \\ e-mail: iwona.dittmann@ue.wroc.pl
}

\begin{abstract}
The aim of the article was to assess the similarities of average price changes in the residential market in 34 European countries in 2010-2016. The first part of the study concerned tendencies of changes in average prices in residential markets in the studied countries, while the second part analyzed cooccurrence of changes in these countries in time. The study covered the period after the first wave of the financial crisis in Europe and took into account the second wave of crisis in several euro area countries. Price indices, trend functions, price ranges, linear correlation coefficients and shape similarity measure were utilized for conducting this study.

European countries, in general, differed with respect to changes in prices in the residential market. 12 countries were characterized by a trend of increasing price indices. 18 countries were classified as correcting countries, as during the studied period they were distinguished by a clear change in trend. Four countries with a downward trend during the study period were also identified. Furthermore, a differentiation between the countries was found due to the values of price ranges during the studied period. Studies of co-occurrence in time were conducted with the use of linear correlation coefficients mainly for groups of rising countries and falling countries. The study was conducted using measures of shape similarity, which allowed for an identification of converging, leading and following markets for some countries.
\end{abstract}

Key words: housing prices, comparative analysis, shape similarity measure.

JEL Classification: R30, R32.

Citation: Dittmann I., 2017, Similarity of Changes in Average Prices of Residential Properties in Europe in 2010-2016, Real Estate Management and Valuation, vol. 25, no. 4, pp. 63-74.

DOI: 10.1515/remav-2017-0030

\section{Introduction}

Monitoring, analysis and forecasting of residential market conditions is essential for national economies. Changes in supply and demand and price changes are significant both at micro-economic scales (for individual household budgets) as well as at a macro scale (stability of the banking sector and national financial stability). Importantly, due to the their long-term nature, these factors may be of strategic importance for a country (due to their connection with demographics). Consequently, research centers in individual countries systematically perform studies concerning the shaping of residential prices (in nominal and real terms), housing availability (i.e. the ratio of housing prices to wages), housing loan availability (taking into count eg. the loan interest), and supply and demand in the housing market. In Poland, apart from scientific centers, such analyses are performed by GUS (Central Statistical Office), NBP (National Bank of Poland), ZBP (Association of Polish Banks), and AMRON-SARFiN. 
Comparative analyses of residential real estate markets are often the topic of research studies. Such comparisons often concern both local real estate markets in a given country as well as real estate markets in different countries. Both comparisons have important scientific implications for real estate market economics. The results obtained have a practical dimension, especially allowing for eg. the diversification of real estate investment fund portfolios, risk management in banks engaged in mortgage activity, and for forecasting residential prices with the use of spatial-temporal analogies.

\section{Literature review}

Analyses of spatial-temporal differentiation in residential real estate markets are most often performed for local markets in a given country, e.g. for the largest cities. From studies of Polish housing markets, mention should be made of in-depth studies concerning pricing dynamics in local markets conducted by CELLMER 2010; BEŁEJ, KULESZA 2015; BEŁEJ 2012 and 2013; TROJANEK 2012 and WOLSKI 2016. Pricing convergence in local markets in Poland was also an object of study (e.g. GNAT 2016; DiTTMANN 2012a and 2014), along with pricing differentiation in local residential markets (eg. ŻELAZOWSKI 2011). Some of the analyses concerned similarities of time series of prices in local housing markets (eg.: BEŁEJ, KULESZA 2014; KRUSZKA, TROJANEK 2014; DiTTMANN 2012a and 2012b).

Among the newest studies of spatial-temporal differentiation of prices in local real estate markets in other countries, attention should be given to a wide-range study of housing price correlation in the biggest cities of the USA conducted by Zimmer (ZIMMER 2015). These studies were dictated by the crisis in housing markets in the USA. By analyzing monthly data, Zimmer has shown that housing prices showed a correlation varying in time, and furthermore this correlation increased during market turmoil. In turn Titman et al. (TITMAN et al., 2014) analyzed housing prices in 97 US metropolitan areas between 1980 and 2011. They showed that annual price changes are characterized by a positive correlation. Miles (MILES 2015) studied regional price segmentation and convergence in US housing markets. Schindler (SCHINDLER 2013) studied persistence of price changes and price predictability in 20 US residential markets in nominal and real terms.

Comparative analyses of price dynamics in real estate markets in different countries are often conducted as part of studies of real estate market cyclicality (among others, AKIMOV et al. 2015; ALVAREZ et al. 2010; BRACKE 2011; DE BANDT et al. 2010; IGAN, LOUNGANI 2012; KYDLAND et al. 2016; ŻELAZOWSKI 2016). The results of these analyses allow for an identification of both similarities and differences in the shaping of long-term trends and to divide countries into groups similar to each other with respect to trends and speculative bubbles (see among others DITTMAR et al. 2015; TSATSARONIS , ZHU 2004; TISSOT 2014). Cesa-Bianchi et al. (CESA-BIANCHI et al. 2015) compared price cycles in housing markets in developed and developing countries (based on quarterly data from 19902012). They have shown that house prices in developing economies rise quicker, are more variable, unstable and are less synchronized than in the case of developed countries. Interesting studies were conducted by Milcheva and Zhu (MiLCHEVA, ZHU 2016). They concerned the convergences of housing prices in developed countries with the banking integration level (as measured by trans-border interbank flows) The researchers found that banking integration levels may capture the level of residential real estate market convergence better than other economic integration measures (eg. international trade, foreign investment, geographical distance etc.). Engsted and Petersen (ENGSTED, PETERSEN 2015) compared 18 OECD countries with respect to the predictability of the degree of return from housing investments and increases in rents with the use of the rent to price ratio as a predictive variable. They have found that 1) for most of the countries studied, the rent to price ratio may to a large degree serve to predict the return from a real estate investment 2) there are large differences between countries and between time periods concerning the predictability of rent increases $\mathrm{w} 3$ ) predictive models depend on the applied approach - nominal or real.

The studies presented in this article concern similarities of 34 European countries with respect to changes in average prices in the residential market (based on price indices). The analysis conducted involved a relatively short study period: 1st quarter 2010 - 2nd quarter 2016. However, this is a unique period, occurring after the first wave of financial crisis in Europe (Ireland, Spain, Portugal and Greece) and including the second wave of crisis in several euro area countries (Ireland, Spain, Greece, Cyprus, Portugal, Italy) Based on the conducted literature review, no answer was found to the question of whether there was a similarity of European countries with respect to changes in housing prices in this "uncertain" period. Conducting a wide-ranging (in terms of the number of countries compared) comparative analysis of the shaping of housing prices partly fills this gap. 
The aim of this article is to evaluate the similarities in changes of average prices in the housing market in European countries during the time period of 2010-2016. The first part of the study deals with changes in average prices in the housing markets in the studied countries, and the second part concerns the co-occurence of these changes in these countries during this time period.

\section{Data and Methodology}

The study was conducted based on 26 quarterly(1st quarter of 2010 - 2nd quarter of 2016) single-base indices $(2010=100)$ of average nominal prices in residential real estate markets in 34 European countries (Austria, Belgium, Bulgaria, Switzerland, Cyprus, Czech Republic, Germany, Denmark, Estonia, Spain, Finland, France, United Kingdom, Greece, Croatia, Hungary, Ireland, Iceland, Italy, Lithuania, Luxembourg, Latvia, Macedonia, Malta, Netherlands, Norway, Poland, Portugal, Romania, Russia, Sweden, Slovenia, Slovak Republic, Turkey). The source of data was the Bank for International Settlements (BIS Residential Property Price database).

In order to perform an analysis of changes in average housing prices, plots of dynamic indices were prepared, trend function parameters were estimated (and the significance of parameters was evaluated) and price index value ranges were calculated for individual countries.

Studies of co-occurrence were conducted in 2 stages. First, Pearson's linear correlation coefficients were calculated. Next, the presence of spatial-temporal analogies was determined with the use of shape similarity measure $m$ (formula 1) (CIEŚLAK, JASIŃSKI 1979; DITTMANN 2012a). Such a determination is conducted as follows. In order to calculate the shape similarity measure, the time series interval for the variable (taken do be the dynamic index) which characterizes the first object (taken to be the studied country) of length $t$ (taken to be the entire studied period) is determined. In the time series describing the second object (another country) a search is made for intervals of the same length $t$ for which the value of the similarity measure as calculated from the formula below is greater than or equal to the assumed threshold value $m^{*}$. By "rolling" the intervals, the researcher has the ability to identify similar objects: convergent (here countries where changes occurred simultaneously), leading (where changes in prices occurred earlier) and following (where changes occurred later).

$$
m=\frac{1}{n-1} \sum_{i=1}^{n-1} m_{i}
$$

where: $\mathrm{m}_{\mathrm{i}}=1-\frac{2}{\pi} \alpha_{i}$, if the signs of slopes of the line passing through points $\left(\mathrm{i}, \mathrm{y}_{\mathrm{i}}\right)$ and $\left(\mathrm{i}+1, \mathrm{y}_{\mathrm{i}+1}\right)$ and the line passing through points $\left(\mathrm{i}, \mathrm{x}_{\mathrm{i}}\right)$ and $\left(\mathrm{i}+1, \mathrm{x}_{\mathrm{i}+1}\right)$ are the same or $\mathrm{m}_{\mathrm{i}}=-\frac{\alpha_{i}}{\pi}$, if the signs of the slopes of the lines are different,

$\alpha_{i}$ - the circular measure of the angle between the two straight lines,

$\mathrm{n}$ - number of observations of $\mathrm{Y}$ and $\mathrm{X}$ variables in the similarity intervals,

$\mathrm{x}_{\mathrm{i}}, \mathrm{x}_{\mathrm{i}+1}$ - the value of the variable characterizing the first object in the period/moment, $\mathrm{i}+1$

$y_{i}, y_{i+1}-$ the value of the variable characterizing the second object in the period/moment, $i+1$

The values of the measure are standardized. There are contained in the range of $\langle-1 ; 1\rangle$. The absolute values of the measure characterizes the degree of similarity (with a positive measure values) or dissimilarity (with a negative measure value) which is higher the closer the value is to unity. The threshold similarity measure value was taken to be $m^{*}=0,6$. In looking for leading and following markets, the series indices were shifted by 1, 2, 3, 4 and 5 quarters.

\section{Empirical results}

\subsection{Similarity of changes in average housing prices - a trend analysis}

Based on a preliminary analysis of price index plots, the studied countries were divided into 3 groups: 1) countries with a trend of increase (rising countries), 2) Countries where a clear change in trend was observed (correcting countries), 3) Countries of a decreasing tendency (falling countries). The price indices are presented in Figures 1-3. The 1st group included: Austria, Belgium, Switzerland, Germany, Estonia, Iceland, Luxembourg, Latvia, Malta, Norway, Sweden and Turkey. The second group included Bulgaria, Denmark, Spain, Finland, France, UK, Hungary, Ireland, Macedonia, the Netherlands, Lithuania, Poland, Portugal, Romania, Russia, Slovenia, Slovakia and the Czech 
Republic. The third group included Cyprus, Greece, Croatia and Italy. For rising countries and falling countries a trend function was estimated (Tab. 1). Calculated p-values for linear trend functions confirmed the correctness of assigning countries to rising and falling groups.

Table 1

Trend functions estimated for rising countries and falling countries

\begin{tabular}{|c|c|c|}
\hline country & trend function & p-value for slope \\
\hline \multicolumn{3}{|c|}{ rising countries } \\
\hline Austria & $Y_{t}=1,7 t+96,67$ & 0.000 \\
\hline Belgium & $Y_{t}=0,39 t+100,72$ & 0.000 \\
\hline Estonia & $Y_{t}=2,83 t+90,46$ & 0.000 \\
\hline Germany & $Y_{t}=0,92 t+96,58$ & 0.000 \\
\hline Iceland & $Y_{t}=2,05 t+92,32$ & 0.000 \\
\hline Latvia & $Y_{t}=1,31 t+100$ & 0.000 \\
\hline Luxembourg & $Y_{t}=1,28 t+95,46$ & 0.000 \\
\hline Malta & $Y_{t}=0,45 t+96,77$ & 0.000 \\
\hline Norway & $Y_{t}=1,47 t+97,9$ & 0.000 \\
\hline Sweden & $Y_{t}=1,86 t+89,23$ & 0.000 \\
\hline Switzerland & $Y_{t}=0,84 t+99,3$ & 0.000 \\
\hline Turkey & $Y_{t}=4,56 t+80,42$ & 0.000 \\
\hline \multicolumn{3}{|c|}{ falling countries } \\
\hline Croatia & $Y_{t}=-0,48 t+102,37$ & 0.000 \\
\hline Cyprus & $Y_{t}=-1,31 t+104,25$ & 0.000 \\
\hline Greece & $Y_{t}=-1,73 t+103,21$ & 0.000 \\
\hline Italy & $Y_{t}=-0,78 t+104,04$ & 0.000 \\
\hline
\end{tabular}

Source: own calculation.

Among the rising countries, there were countries with very low index values i.e. Malta, Belgium (approximately 110 at the end of the studied period) as well as countries with exceptionally high index values at the end of the studied period, i.e. Turkey (about 210) and Estonia (approximately 160). Price indices of other countries at the end of the studied period ranged from approximately 120 (Switzerland) to approximately 150 (Iceland). The correcting countries group was very varied with respect to both the direction of trend change as well as index values. Among these countries there were countries with an initially constant trend that then changed into an increasing one. This small subgroup is therefore rather similar to rising countries. These countries are the UK, Czech Republic and Lithuania. The second, more numerous subgroup is characterized by an approximately parabolic shape of the trend function, where after a period of decrease a period of increase occurred. In this subgroup, the following can be distinguished: a) countries of low price decreases $(\max 10 \%)$ as compared to 2010 (baseline) in the first period, where at the end of the studied period the prices exceeded those from 2010: Denmark, Hungary, Slovakia, b) countries where low initial price decreases (max 15\%) occurred, where at the end of the studied period the prices reached 2010 levels or were slightly lower: Bulgaria, Portugal, Poland, Macedonia, the Netherlands, c) countries with large initial decreases (15\%-30\%) and a small later correction: Spain, Romania, Slovenia, d) a country with a large decrease followed by a large increase: Ireland. The last sub-group of correcting countries is made up of 3 countries: France, which at first was characterized by an increasing tendency followed by a decreasing trend, Finland, where first an increase occurred followed by price stabilization, and Russia, where first a large decrease was observed, followed by a return to 2010 levels, after which another decrease occurred. The falling countries group is small, with four countries: Greece (the price index value at the end of the studied period equal to about 64), cyprus (approximately 74), Italy(approximately 85) and Croatia (about 90). 


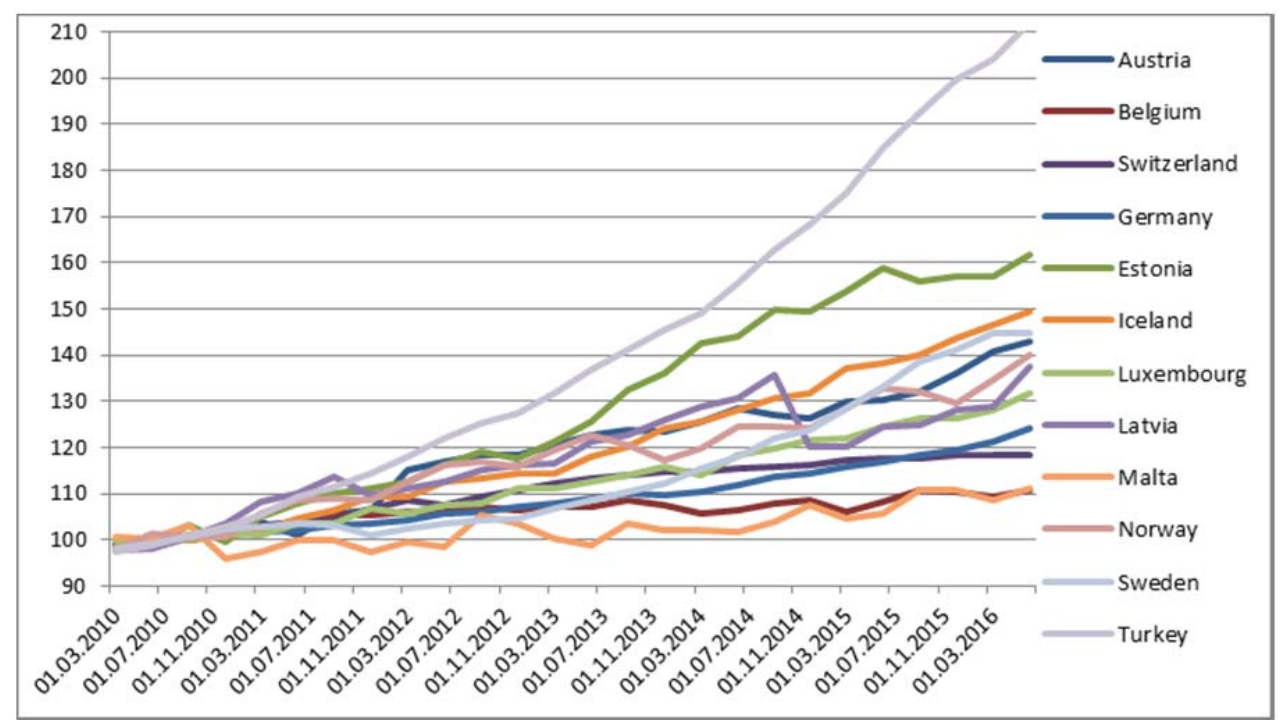

Fig. 1. Single-base indices $(2010=100)$ of average nominal prices - rising countries. Source: own elaboration basing on BIS Residential Property Price database.

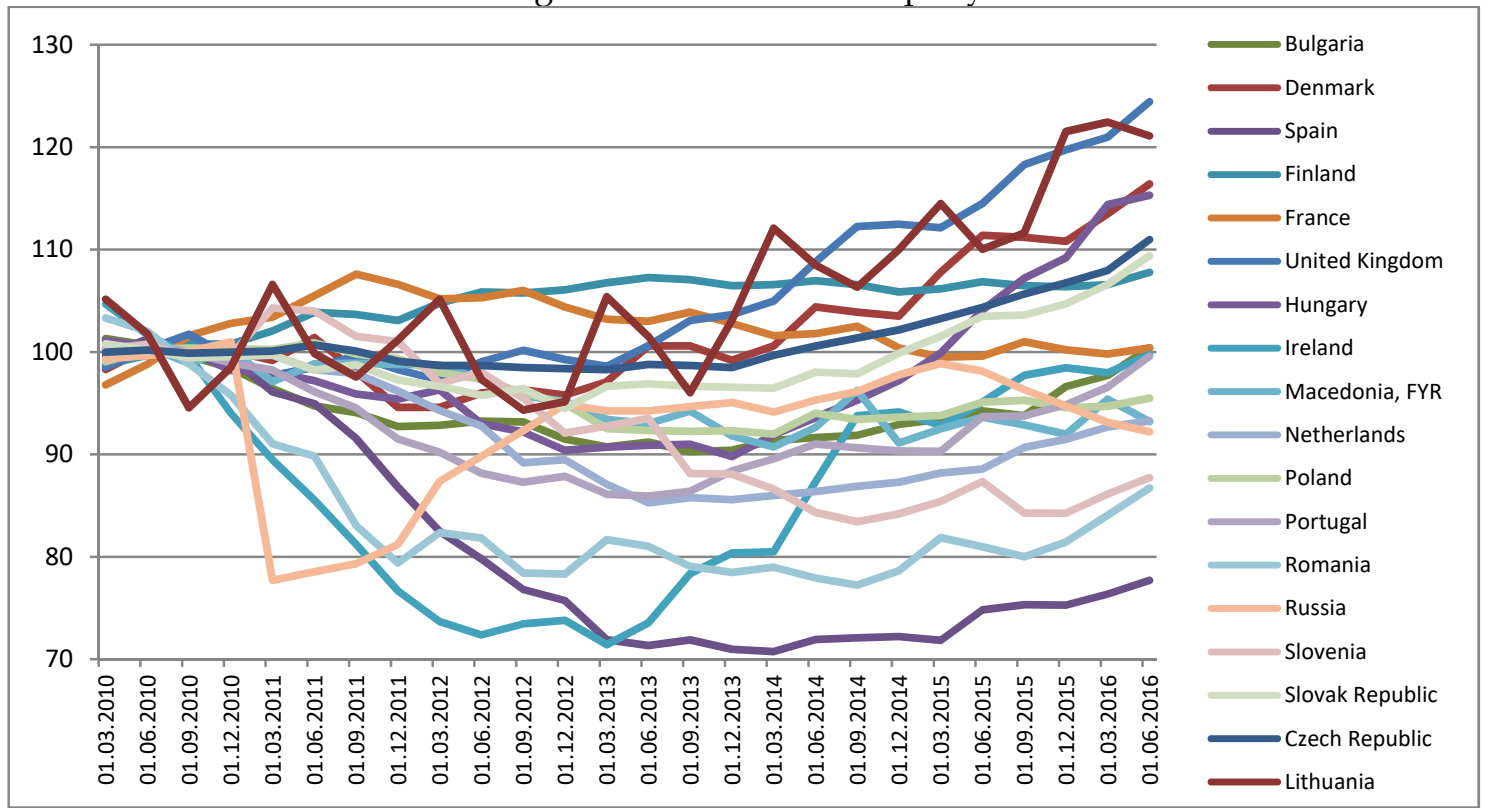

Fig. 2. Single-base indices $(2010=100)$ of average nominal prices - correcting countries. Source: own elaboration basing on BIS Residential Property Price database.

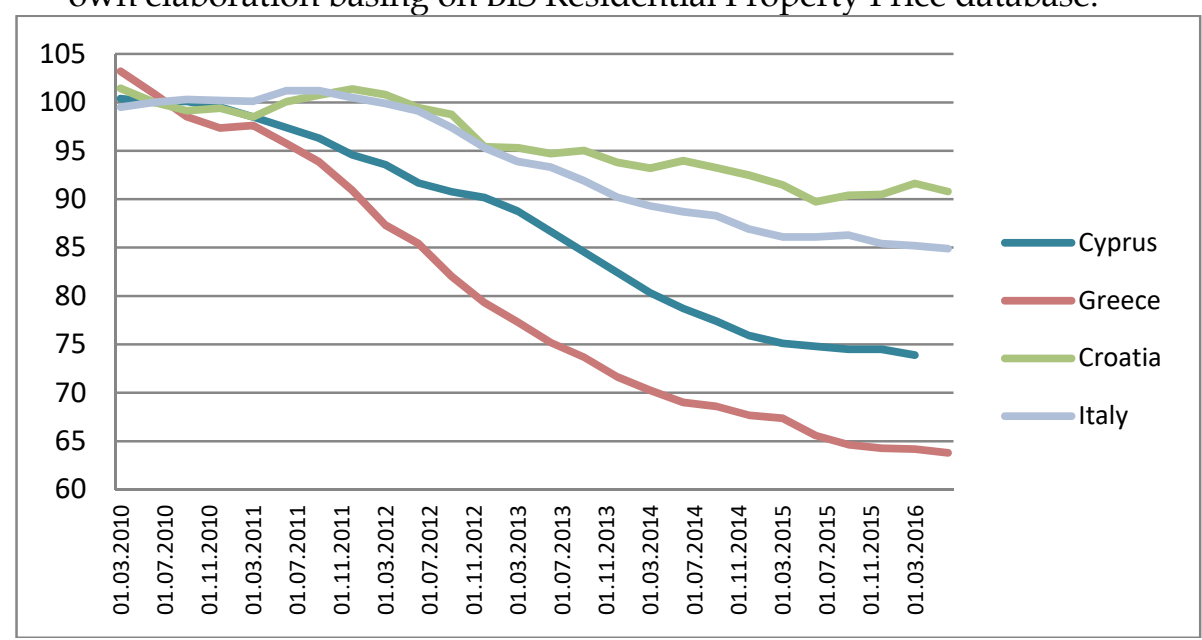

Fig. 3. Single-base indices $(2010=100)$ of average nominal prices - falling countries. Source: own elaboration basing on BIS Residential Property Price database. 
A comparison of countries was also made with respect to the lowest and highest dynamic index values in the studied period, and the dynamic index value range in the studied period (Fig. 4). It is possible to distinguish countries with the highest index values of 100 or slightly higher (Greece, Spain, Ireland, Cyprus, Romania, Russia, Slovenia, Italy, Netherlands, Portugal, Croatia, Bulgaria, Macedonia, Poland) and countries with the lowest index values (Austria, Finland, Germany, Luxembourg, Iceland, Estonia, Switzerland, Czech Republic, Belgium, Sweden, Latvia, Norway, UK, Turkey, France, Malta, Denmark, Slovakia and Lithuania). In the case of Spain, the lowest index value was approximately 90 and the highest value was approx. 115. Among the studied countries it is possible to indicate both countries with small range values of about 9-12 percent (Poland, Finland, Macedonia, France, Bulgaria, Croatia), countries with average range values of about 13-23 percent, Belgium, Czech Republic, Portugal, Malta, Netherlands, Slovakia, Italy, Switzerland, Slovenia, Denmark, Russia), countries with high range values, about 25-40 percent (Germany, Hungary, Romania, Cyprus, UK, Lithuania, Spain, Luxembourg, Ireland, Greece, Latvia, Norway, Austria) and very large (Sweden, Iceland, Estonia, Turkey).

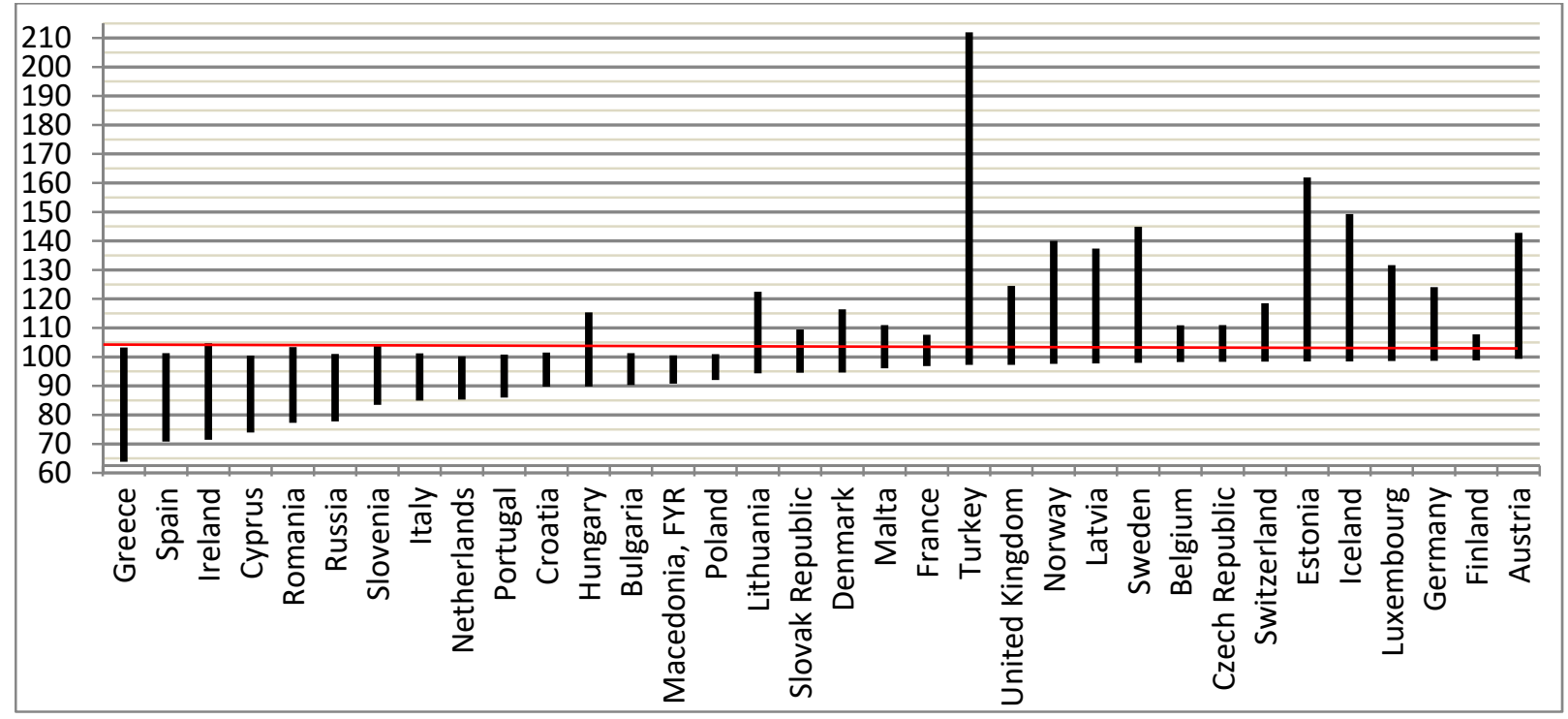

Fig. 4. The lowest and highest dynamic index values for individual countries. Source: own elaboration basing on BIS Residential Property Price database.

\subsection{Co-occurrence of price changes over time}

In order to evaluate the co-occurrence of price changes in time in the studied markets, linear correlation coefficients of price indices were calculated (Tab. 2). The following groups of countries were identified: 1) With a high positive correlation of dynamic indices (coefficient value > 0.8), 2) With a high negative correlation of dynamic indices, 3) with low absolute values of correlation coefficients or statistically insignificant values. High positive correlation coefficients characterized : 1) almost the entire rising countries group: Austria, Belgium, Switzerland, Germany, Estonia, Iceland, Luxembourg, Latvia, Norway, Turkey; 2) the entire group of falling countries; 3) some countries in the correcting countries group (eg. Bulgaria and Portugal, Poland and the Netherlands, Poland and Spain, Hungary, the Czech Republic and Slovakia). High negative correlation coefficient values were observed mainly between rising countries and falling countries, but also between rising countries and correcting countries. (eg. Austria and Poland), and between falling countries and correcting countries (eg. Greece and Finland) and between countries in the correcting countries group (eg. Finland and Spain).

Values of correlation coefficients

Table 2

\begin{tabular}{|c|c|c|c|c|c|c|c|c|c|c|c|c|c|c|c|c|c|}
\hline kraje & $\mathrm{AU}$ & $\mathrm{BE}$ & $\mathrm{BU}$ & SW & $\mathrm{CY}$ & $\mathrm{CZ}$ & GE & $\mathrm{DE}$ & ES & SP & $\mathrm{FI}$ & FR & UK & GR & CR & $\mathrm{HU}$ & IR \\
\hline $\mathrm{AU}$ & 1 & .88 & -.49 & .98 & -.96 & .58 & .96 & .66 & .95 & -.89 & .87 & -.26 & .81 & -.98 & -.90 & .25 & -.02 \\
\hline $\mathrm{BE}$ & .88 & 1 & -.66 & .91 & -.83 & .43 & .85 & .46 & .82 & -.86 & .91 & 1 & 64 & -.89 & -.74 & 0 & .27 \\
\hline $\mathrm{BU}$ & -.49 & -.66 & 1 & \begin{tabular}{|c|}
-.61 \\
\end{tabular} & .44 & 31 & -.34 & .18 & -.40 & .80 & -.82 & -.4 & .01 & .57 & .34 & 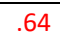 & 75 \\
\hline SW & .98 & .91 & -.61 & $\perp$ & .97 & .51 & .95 & .61 & .95 & -.92 & .91 & -.18 & .77 & -.99 & -.91 & .14 & -.08 \\
\hline $\mathrm{CY}$ & -.96 & -.83 & .44 & -.97 & 1 & -.64 & -.98 & -.74 & -.99 & .84 & -.80 & .36 & -.88 & .98 & .94 & -.29 & -.14 \\
\hline
\end{tabular}




\begin{tabular}{|c|c|c|c|c|c|c|c|c|c|c|c|c|c|c|c|c|c|}
\hline & 58 & .43 & 31 & 51 & .64 & 1 & .75 & .93 & .69 & -.17 & .21 & -.54 & .91 & $\mid-.52$ & -.65 & .90 & .66 \\
\hline GE & 96 & .85 & -.34 & 95 & -.98 & .75 & 1 & .80 & .98 & $\begin{array}{l}-78 \\
\end{array}$ & .77 & -.34 & .92 & \begin{tabular}{|l|}
-.95 \\
\end{tabular} & -.93 & .43 & 10 \\
\hline $\mathrm{DE}$ & 56 & 46 & 18 & 61 & 74 & .93 & .8 & 1 & 78 & 30 & .33 & -.61 & 93 & -.64 & -.79 & .77 & 65 \\
\hline$\overline{E S}$ & 95 & .82 & 40 & 95 & -.99 & .69 & .98 & .78 & 1 & -.80 & .77 & -.37 & .90 & -.96 & -.94 & .35 & 19 \\
\hline SP & 89 & -.86 & 80 & 92 & 84 & -.17 & -.78 & 30 & -.8 & 1 & -.95 & .01 & .50 & .92 & .76 & .20 & 38 \\
\hline $\mathrm{FI}$ & 87 & .91 & .82 & 91 & -.80 & .21 & 77 & 33 & 77 & .95 & 1 & 16 & .48 & -.89 & -.73 & $\begin{array}{l}-.17 \\
\end{array}$ & .44 \\
\hline $\mathrm{FR}$ & 26 & .13 & .49 & .18 & .36 & -.54 & -.34 & .61 & .37 & .01 & .16 & 1 & -.54 & .24 & .47 & $\begin{array}{l}-.56 \\
\end{array}$ & -.78 \\
\hline UK & .81 & .64 & 01 & 77 & -.88 & .91 & .92 & 93 & .90 & -.50 & .48 & 54 & 1 & \begin{tabular}{|c|}
.79 \\
\end{tabular} & -.86 & .68 & 53 \\
\hline GR & \begin{tabular}{|c|}
-.98 \\
\end{tabular} & .89 & 57 & -.99 & 98 & .52 & -.95 & -.64 & .96 & .92 & \begin{tabular}{|l|}
-89 \\
\end{tabular} & .24 & .79 & 1 & .93 & $\begin{array}{l}-.16 \\
\end{array}$ & .03 \\
\hline CR & \begin{tabular}{|c|}
-.90 \\
\end{tabular} & \begin{tabular}{|l|}
-.74 \\
\end{tabular} & 34 & -.91 & .94 & $\begin{array}{l}-.65 \\
\end{array}$ & -.93 & -.79 & \begin{tabular}{|l|}
-.94 \\
\end{tabular} & .76 & -.73 & .47 & -.86 & \begin{tabular}{|l|}
93 \\
\end{tabular} & 1 & $\begin{array}{l}-.32 \\
\end{array}$ & -.23 \\
\hline $\mathrm{HU}$ & 25 & .10 & 64 & 14 & \begin{tabular}{|c|}
-.29 \\
\end{tabular} & .90 & .43 & .77 & .35 & .20 & -.17 & -.56 & .68 & -.16 & -.32 & 1 & .76 \\
\hline IR & -.02 & $\begin{array}{l}-.27 \\
\end{array}$ & .75 & -.08 & -.14 & .66 & .18 & 65 & 19 & .38 & -.44 & --.78 & .53 & .03 & -.23 & .76 & 1 \\
\hline IC & 96 & .83 & -.34 & 95 & -.98 & .75 & .99 & 80 & .99 & \begin{tabular}{l|l}
-.78 \\
\end{tabular} & .76 & \begin{tabular}{|l|}
-.37 \\
\end{tabular} & .92 & -.95 & \begin{tabular}{|l|}
-.93 \\
\end{tabular} & .43 & .20 \\
\hline IT & 93 & -.74 & 33 & -.93 & 98 & -.67 & -.95 & -.78 & -.97 & .78 & -.72 & .51 & -.89 & .95 & .97 & -.34 & -.26 \\
\hline L & .67 & .42 & .09 & .62 & -.71 & .82 & .75 & .77 & .73 & -.37 & .33 & -.60 & .80 & -.62 & -.69 & .67 & .46 \\
\hline LU & 96 & .86 & -.39 & 96 & -.98 & .71 & .99 & .78 & .98 & -.80 & .78 & -.35 & .90 & -.96 & -.94 & .39 & .16 \\
\hline LA & .90 & .85 & -.64 & 93 & -.90 & .42 & .86 & .54 & .89 & -.87 & .89 & -.06 & .69 & -.93 & -.82 & .04 & -.11 \\
\hline $\mathrm{MC}$ & -.83 & -.75 & .62 & -.87 & .84 & -.29 & -.77 &.- .44 & \begin{tabular}{|l|}
-.81 \\
\end{tabular} & .88 & -.82 & .22 & -.58 & .88 & .86 & .08 & .14 \\
\hline $\mathrm{ML}$ & .73 & .65 & -.03 & .68 & -.76 & .75 & .81 & .75 & .78 & -.50 & .47 & -.43 & .86 & -.72 & -.76 & .56 & .40 \\
\hline $\mathrm{NE}$ & -.80 & $\begin{array}{l}-.76 \\
\end{array}$ & .82 & -.85 & .77 & \begin{tabular}{|l|}
-.03 \\
\end{tabular} & -.67 & -.22 & -.72 & .97 & -.90 & .03 & -.39 & .86 & .72 & .35 & .39 \\
\hline $\mathrm{NO}$ & .97 & .90 & -.50 & .97 & -.96 & .62 & .97 & 70 & .95 & -.86 & .88 & -.20 & .81 & -.96 & -.90 & .28 & -.02 \\
\hline $\mathrm{PL}$ & -.83 & -.72 & .69 & -.85 & .79 & -.13 & -.72 & -.30 & -.75 & .94 & -.84 & .20 & -.47 & .87 & .77 & .22 & .26 \\
\hline $\mathrm{PO}$ & \begin{tabular}{l|l|}
-.51 \\
\end{tabular} & \begin{tabular}{|c|}
-.64 \\
\end{tabular} & .93 & -.58 & .40 & .35 & -.32 & 25 & -.35 & .83 & -.80 & -.42 & .05 & .55 & .30 & .64 & .80 \\
\hline RO & -.73 & -.87 & .89 & -.81 & .69 & -.06 & -.63 & -.11 & -.66 & .89 & -.92 & -.38 & -.34 & .77 & .54 & .30 & .56 \\
\hline $\mathrm{RU}$ & .30 & .02 & .18 & .24 & -.34 & .20 & .29 & 35 & .32 & -.22 & .03 & -.71 & .38 & \begin{tabular}{|l|}
-.32 \\
\end{tabular} & -.44 & .15 & .43 \\
\hline SE & .87 & .73 & -.10 & .83 & -.91 & .90 & 96 & 92 & .93 & -.58 & .59 & -.47 & .98 & \begin{tabular}{|l|}
-.84 \\
\end{tabular} & -.89 & .65 & .40 \\
\hline$S L$ & -.92 & -.75 & 44 & -.92 & 95 & -.51 & -.90 & -.64 & -.93 & .84 & -.75 & .41 & -.81 & .95 & .91 & -.17 & -.15 \\
\hline SR & .34 & .16 & .56 & .25 & -.41 & .93 & .53 & .86 & .46 & .10 & -.08 & -.64 & .76 & -.27 & -.46 & .96 & .79 \\
\hline TU & .94 & .82 & -.28 & .93 & -.97 & .79 & .99 & .84 & .98 & -.73 & .72 & -.40 & .94 & -.93 & -.93 & .49 & .25 \\
\hline
\end{tabular}

AU - Austria, BE - Belgium, BU - Bulgaria, SW - Switzerland, CY - Cyprus, CZ - Czech Republic, GE - Germany, DE - Denmark, ES Estonia, SP - Spain, FI - Finland, FR - France, UK - United Kingdom, GR - Greece, CR - Croatia, HU - Hungary, IR - Ireland, IC Iceland, IT - Italy, LI - Lithuania, LU - Luxembourg, LA - Latvia, MC - Macedonia, ML - Malta, NE - Netherlands, NO - Norway, PL Poland, PO - Portugal, $\quad$ RO - Romania, RU - Russia, SE - Sweden, SL - Slovenia, SR - Slovak Republic, TU - Turkey

Source: own calculation.

Values of correlation coefficients

Table 2 cd.

\begin{tabular}{|c|c|c|c|c|c|c|c|c|c|c|c|c|c|c|c|c|c|}
\hline raje & IC & IT & LI & LU & LA & $C$ & $\mathrm{ML}$ & $\mathrm{NE}$ & $\mathrm{NO}$ & $\mathrm{PL}$ & \begin{tabular}{|l|} 
PO \\
\end{tabular} & RO & $\mathrm{RU}$ & SE & $\overline{S L}$ & SR & TU \\
\hline $\mathrm{AU}$ & .96 & 3 & 67 & 96 & 90 & -.83 & 73 & -.80 & .97 & -.83 & -.51 & -.73 & 30 & 87 & -.92 & .34 & 94 \\
\hline $\mathrm{BE}$ & 33 & 74 & 42 & 86 & 35 & -.75 & .65 & -.76 & 90 & -.72 & -.64 & -.87 & 02 & 73 & 75 & $\overline{16}$ & 82 \\
\hline$\overline{B U}$ & \begin{tabular}{|l|} 
\\
\end{tabular} & 33 & .09 & 39 & \begin{tabular}{|l|}
-.64 \\
\end{tabular} & .62 & -.03 & 82 & .50 & 69 & 93 & 89 & 18 & .10 & 44 & .56 & .28 \\
\hline SW & 95 & 93 & 62 & 96 & 93 & -.87 & 68 & -.85 & 97 & -.85 & -.58 & -.81 & 24 & 83 & -.92 & .25 & 93 \\
\hline$\overline{C Y}$ & -.98 & 98 & 71 & 98 & \begin{tabular}{|c|}
-.90 \\
\end{tabular} & 84 & -.76 & .77 & \begin{tabular}{l|}
-.96 \\
\end{tabular} & 79 & 40 & 69 & -.34 & \begin{tabular}{|l|}
-91 \\
\end{tabular} & 95 & .41 & .97 \\
\hline CZ & 75 & 67 & 82 & 71 & 42 & -.29 & .75 & -.03 & .62 & -.13 & 35 & $\begin{array}{l}-.06 \\
\end{array}$ & 20 & 90 & -.51 & .93 & 79 \\
\hline GE & 9 & -.95 & 75 & 99 & 86 & \begin{tabular}{|c|}
-.77 \\
\end{tabular} & .81 & -.67 & 97 & -.72 & -.32 & -.63 & 29 & 96 & -.90 & 53 & 99 \\
\hline$\overline{\mathrm{DE}}$ & 80 & .78 & 77 & 78 & 4 & -.44 & .75 & .22 & 70 & -.30 & .25 & -.1 & 35 & 92 & .64 & 86 & 84 \\
\hline $\mathrm{ES}$ & .99 & -.97 & 73 & 98 & 39 & -.81 & .78 & -.72 & 95 & -.75 & -.35 & -.66 & 32 & 93 & -.9 & 46 & 98 \\
\hline SP & -.78 & 78 & 37 & -.8 & -.87 & .88 & -.50 & .97 & -.86 & .94 & 83 & 89 & .22 & -.58 & 84 & 10 & .73 \\
\hline $\mathrm{FI}$ & 76 & -.72 & 33 & 78 & 89 & -82 & .47 & -.90 & 88 & -.84 & -.80 & -.92 & 03 & 59 & -.75 & .08 & 72 \\
\hline FR & -.37 & .51 & .60 & .35 & -.06 & .22 & -.43 & .03 & -.20 & 20 & -.42 & -.38 & .71 & -.47 & 41 & .64 & .40 \\
\hline UK & .92 & -.89 & 80 & .90 & .69 & -.58 & .86 & -.39 & .81 & -.47 & .05 & -.34 & 38 & 98 & -81 & 76 & 94 \\
\hline GR & -.95 & 95 & -.62 & $\begin{array}{l}-.96 \\
\end{array}$ & $\begin{array}{l}-93 \\
\end{array}$ & .88 & -.72 & .86 & $\begin{array}{l}-.96 \\
\end{array}$ & 87 & .55 & 77 & $\begin{array}{l}-32 \\
\end{array}$ & -.84 & 95 & -.27 & $\begin{array}{l}-.93 \\
\end{array}$ \\
\hline$C R$ & -.93 & .97 & -.69 & -.94 & -.82 & .86 & -.76 & 72 & -.90 & 77 & .30 & .54 & -.44 & -.89 & 91 & -.46 & -.93 \\
\hline $\mathrm{HU}$ & .43 & \begin{tabular}{|l|} 
\\
\end{tabular} & .67 & .39 & .04 & .08 & .56 & .35 & .28 & .22 & .64 & .30 & .15 & .65 & -.17 & .96 & .49 \\
\hline IR & .20 & -.26 & .46 & .16 & \begin{tabular}{l|}
-.11 \\
\end{tabular} & .14 & .40 & .39 & $\begin{array}{l}-02 \\
\end{array}$ & .26 & .80 & .56 & .43 & .40 & -.15 & .79 & .25 \\
\hline IC & 1 & -.96 & 76 & .99 & .86 & -.78 & .80 & -.67 & .96 & -.71 & -.31 & -.63 & 30 & .96 & -.91 & .52 & .99 \\
\hline IT & -.96 & 1 & -.74 & 96 & -.85 & .84 & -.77 & .73 & -.91 & .80 & .31 & .56 & -.46 & -.91 & .96 & -.47 & .95 \\
\hline $\mathrm{LI}$ & 76 & -.74 & 1 & 73 & .54 & -.51 & .58 & -.25 & .65 & -.38 & 13 & -.19 & .17 & .83 & -.61 & .73 & 79 \\
\hline LU & .99 & -.96 & 73 & 1 & .87 & \begin{tabular}{|c|}
-79 \\
\end{tabular} & .79 & -.70 & .96 & -.74 & -.36 & -.66 & 31 & .94 & -.92 & 49 & 99 \\
\hline$\overline{L A}$ & 86 & -.85 & .54 & .87 & 1 & -80 & .57 & -.83 & 88 & -.82 & -.55 & -.82 & 12 & 74 & -.87 & 14 & 83 \\
\hline $\mathrm{MC}$ & -.78 & .84 & -.51 & \begin{tabular}{|c|}
-.79 \\
\end{tabular} & -.80 & 1 & -.57 & .87 & -.80 & .89 & .60 & .71 & -.28 & -.64 & .83 & -.08 & .75 \\
\hline $\mathrm{ML}$ & 80 & -.77 & .58 & .79 & .57 & -.57 & 1 & -.40 & .72 & -.47 & -.06 & -.35 & 39 & 84 & -.75 & 59 & 83 \\
\hline $\mathrm{NE}$ & -.67 & .73 & -.25 & -.70 & -.83 & .87 & -.40 & 1 & -.77 & .96 & 84 & .83 & -.30 & -.46 & .81 & 21 & -.62 \\
\hline NO & 96 & -.91 & 65 & .96 & 88 & -.80 & .72 & -.77 & 1 & -.77 & -.49 & -.74 & 21 & 88 & -.87 & 37 & 95 \\
\hline$\overline{P L}$ & \begin{tabular}{|c|}
-.71 \\
\end{tabular} & .80 & -.38 & -.74 & -.82 & .89 & -.47 & .96 & \begin{tabular}{l|}
-.77 \\
\end{tabular} & 1 & .74 & .72 & -.40 & -.53 & .85 & .08 & -.67 \\
\hline
\end{tabular}




\begin{tabular}{|l|r|r|r|r|r|r|r|r|r|r|r|r|r|r|r|r|r|} 
PO & -.31 & .31 & .13 & -.36 & -.55 & .60 & -.06 & .84 & -.49 & .74 & 1 & .84 & .02 & -.06 & .43 & .58 & -.25 \\
\hline RO & -.63 & .56 & -.19 & -.66 & -.82 & .71 & -.35 & .83 & -.74 & .72 & .84 & 1 & .14 & -.43 & .63 & .24 & -.58 \\
\hline RU & .30 & -.46 & .17 & .31 & .12 & -.28 & .39 & -.30 & .21 & -.40 & .02 & .14 & 1 & .3 & -.52 & .22 & .31 \\
\hline SE & .96 & -.91 & .83 & .94 & .74 & -.64 & .84 & -.46 & .88 & -.53 & -.06 & -.43 & .30 & 1 & -.82 & .73 & .98 \\
\hline SL & -.91 & .96 & -.61 & -.92 & -.87 & .83 & -.75 & .81 & -.87 & .85 & .43 & .63 & -.52 & -.82 & 1 & -.29 & -.89 \\
\hline SR & .52 & -.47 & .73 & .49 & .14 & -.08 & .59 & .21 & .37 & .08 & .58 & .24 & .22 & .73 & -.29 & 1 & .59 \\
\hline TU & .99 & -.95 & .79 & .99 & .83 & -.75 & .83 & -.62 & .95 & -.67 & -.25 & -.58 & .31 & .98 & -.89 & .59 & 1 \\
\hline
\end{tabular}

$\mathrm{AU}$ - Austria, BE - Belgium, BU - Bulgaria, SW - Switzerland, CY - Cyprus, CZ - Czech Republic, GE - Germany, DE - Denmark, ES Estonia, SP - Spain, FI - Finland, FR - France, UK - United Kingdom, GR - Greece, CR - Croatia, HU - Hungary, IR - Ireland, IC Iceland, IT -Italy, LI - Lithuania, LU - Luxembourg, LA - Latvia, MC - Macedonia, ML - Malta, NE - Netherlands, NO - Norway, PL Poland, PO - Portugal, $\quad$ RO - Romania, RU - Russia, SE - Sweden, SL - Slovenia, SR - Slovak Republic, TU - Turkey

\section{Source: own calculation.}

In the next step, the presence of spatial-temporal analogies was investigated with the use of shape similarity measures ${ }^{1}$. Table 3 presents converging, leading and following with respect to each of the studied countries. In parentheses, the values of shape similarity measures exceeding the threshold value of $\mathrm{m}^{*}=0,6$ is given, along with the number of quarters of lag (in the case of following countries) or advance (in the case of leading markets) ${ }^{2}$. Among the studied countries, the following groups of countries may be identified where a) several leading and/or following and/or converging markets were identified (Germany, Estonia, Iceland, Sweden, Turkey, Luxembourg and Latvia), b) 1-2 leading, following or converging markets were identified (Austria, Belgium, Switzerland, Spain, Finland, UK, Greece, Ireland, Italy, Portugal) and c) where no converging, leading or following markets were identified (countries not mentioned above) The vast majority of spatial-temporal analogies were observed among rising countries. Furthermore, three cases of analogies between rising and correcting country groups were identified (Belgium and Finland, Belgium and France, UK and Sweden), one case of an analogy between falling and correcting countries (Italy and Portugal) and one case of an analogy between falling countries (Greece and Italy). It must be noted that the measure values are not high, with almost all of them being in the range between 0.6 and 0.7 , and therefore the similarity of price shaping in the studied markets as measured by the shape similarity measure is not high, both when assuming no shift in time and when taking into account the possibility of lags in changes.

Table 3

Shape similarity measures and shifts

\begin{tabular}{|c|c|c|c|}
\hline Country & Leading country & $\begin{array}{c}\text { Converging } \\
\text { country }\end{array}$ & Following country \\
\hline Austria & Iceland ( 3 quarters, 0.67 ) & $\begin{array}{c}\text { Germany } \\
(0.61)\end{array}$ & - \\
\hline Belgium & Finland (1 quarter, 0.62) & France $(0,61)$ & - \\
\hline Bulgaria & - & - & - \\
\hline Switzerland & Germany (1 quarter, 0.71 ) & - & Iceland (2 quarters, 0.601$)$ \\
\hline Czech Republic & - & - & - \\
\hline Germany & $\begin{array}{c}\text { Estonia (4 quarters, } 0.63), \text { Sweden }(1 \\
\text { quarter, } 0.66), \text { Turkey (5 quarters, } \\
0.64)\end{array}$ & Austria (0.61) & $\begin{array}{c}\text { Iceland (1 quarter, } 0.7) \text {, Luxembourg (1 } \\
\text { quarter, } 0.63), \text { Switzerland (1 quarter, } \\
0.71)\end{array}$ \\
\hline Denmark & - & - & - \\
\hline Estonia & - & - & $\begin{array}{c}\text { Luxembourg (5 quarters, } 0.67) \text {, Turkey } \\
\text { (4 quarters, } 0.67) \text {, Sweden (4 quarters, } \\
0.63) \text {, Germany (4 quarters, } 0.63 \text { ), Iceland } \\
\text { (5 quarters, } 0.62)\end{array}$ \\
\hline Spain & Ireland (3 quarters, 0.64 ) & - & - \\
\hline Finland & - & - & Belgium (1 quarter, 0.62) \\
\hline France & - & - & - \\
\hline United Kingdom & Sweden (5 quarters, 0.601 ) & - & - \\
\hline Greece & - & - & Italy (1 quarter, 0.64 ) \\
\hline
\end{tabular}

\footnotetext{
1 To perform these calculations, a program by J. Szanduła was used (SZANDUŁA).

2 In the case when for a pair of countries being compared the measure value $m$ was greater than 0.6 more than once. i.e. for several time shifts $\mathrm{m}>0.6$, the table presents only the time shift with the highest measure value.
} 


\begin{tabular}{cccc}
\hline Croatia & - & - & - \\
\hline Hungary & - & - & - \\
\hline Ireland & - & - & Spain (3 quarters, 0.64) \\
Iceland & $\begin{array}{c}\text { Switzerland (2 quarters, 0.601), } \\
\text { Germany (1 quarter, 0.7), Estonia (5 } \\
\text { quarters, 0.62), Sweden (2 quarters, } \\
0.71), \text { Turkey (5 quarters, 0.80) }\end{array}$ & - & Latvia (5 quarters, 0.62), Austria (3 \\
& quarters, 0.67)
\end{tabular}

\begin{tabular}{|c|c|c|c|}
\hline Latvia & $\begin{array}{c}\text { Iceland (5 quarters, } 0.62) \text {, Sweden (1 } \\
\text { quarter, } 0.63) \text {, Turkey (1 quarter, } \\
0.65)\end{array}$ & - & - \\
\hline Macedonia, FYR & - & - & - \\
\hline Malta & - & - & - \\
\hline Netherlands & - & - & - \\
\hline Norway & - & - & - \\
\hline Poland & - & - & - \\
\hline Portugal & - & - & Italy (5 quarters, 0.61 ) \\
\hline Romania & - & - & - \\
\hline Russia & - & - & - \\
\hline Sweden & Estonia (4 quarters, 0.63 ) & - & $\begin{array}{l}\text { Turkey (1 quarter, 0.69), Germany (1 } \\
\text { quarter, 0.66), Iceland (2 quarter, 0.71), } \\
\text { Latvia (1 quarter, 0.63), UK (5 quarters, } \\
0.601) \text {, Luxembourg (1 quarter, 0.605) }\end{array}$ \\
\hline Slovenia & - & - & - \\
\hline Slovak Republic & - & - & - \\
\hline Turkey & $\begin{array}{c}\text { Estonia (4 quarters, } 0.67) \text {, Sweden (1 } \\
\text { quarter, } 0.69)\end{array}$ & - & $\begin{array}{l}\text { Iceland ( } 5 \text { quarters, } 0.80) \text {, Germany ( } 5 \\
\text { quarters, } 0.64) ; \text { Latvia ( } 1 \text { quarter, } 0.65)\end{array}$ \\
\hline
\end{tabular}

Source: own calculation.

\section{Discussion and conclusions}

European countries generally differed in terms of price changes in the housing market. However, it was possible to divide them into groups and subgorups with common characteristics. 12 countries were characterized by an increasing price index trend. However the rate of increase was different. 18 countries were classified as correcting countries, as they were characterized by a clear change in trend during the study period. In the case of most countries from the correcting countries group the prices first decreased and then rose. However, significant differences in the amounts of decrease and increase were observed. In the case of some countries, a period of price stabilization was observed instead of an increase or decrease phase. Furthermore, there are countries with typical (as compared with other countries) trends, eg. an increase followed by a decrease (France) or multiple changes in trend (Russia). Furthermore, 4 countries with a decreasing tendency during the study period were found. Additionally, a differentiation of countries with respect to the price index range during the studied period was found. Countries were divided into three distinct groups: with small range values, (approximately 9-12 percent), average range values(approximately 14-23 percent) and large and very large range values.

A study of co-occurrence of changes in time conducted with the use of linear correlation coefficients have shown the existence of correlations mainly in rising and falling country groups. Interestingly, in the case of Poland a high correlation coefficient was found in relation to the Netherlands and Spain. On the other hand, the Czech Republic, Slovakia and Hungary (the remaining Vysehrad group countries) represented a distinct group, also with high correlation coefficients. The studies were performed utilizing a shape similarity measure, which allowed for the identification of converging, leading and following markets for some (not all) countries. However, it must be noted 
that the values of measures were not high. This indicates that the shape similarity was not large. A similar study performed for the eight largest cities in Poland in the time period from the 1st quarter of 2006 to the 2nd quarter of 2011 yielded much more interesting results in the form of large similarity measure values (DITTMANN 2012a). Therefore, it may be concluded that factors connected with the economy of a given country are of great importance to the shaping of real estate market price changes. A similar conclusion can be drawn from the conducted trend analysis. The presence of many countries with an increasing tendency, several countries characterized by a varying trend and some countries of a decreasing tendency indicate a large role of national factors in determining prices in the residential real estate market. Of course, this does not exclude the existence of connections in the form of, for example, unified credit policies of mortgage banks.

No similarity of countries with respect to price change was observed when separating countries into developing and developed ones. Both in the developing and developed country groups there were countries (residential markets) identified with rising, varying and decreasing trends. It was also noted that the highest increases were recorded in Turkey and Estonia - developing countries. Furthermore, the division of countries into euro area and non-euro area countries was not reflected in price change tendencies and the size of changes.

The occurrence of differences in price trends in real estate markets, in particular the existence of pairs of countries with negative linear correlation coefficients, indicates a possibility of portfolio diversification per Markowitz's portfolio theory. The presence of countries with both low and high price dynamics allows for real estate portfolio diversification with respect to varied investment risk levels.

It is important to highlight the difficulties of conducting research on real estate price dynamics, especially across different countries. The problems connected with conducting a comparative analysis of real estate markets in different countries using statistical methods are shown by, among others, HORSEWOOD 2011, SCATIGNA et al. 2014, STEINER 2013. To a large degree these difficulties result from different data collection systems in different countries and different degrees of data aggregation.

\section{References}

AKIMOV A., STEVENSON S., YOUNG J., 2015, Synchronisation and commonalities in metropolitan housing market cycles, Urban Studies, vol. 52, issue 9, p. 1665-1682, http://dx.doi.org/10.1177 /0042098014535643.

Alvarez L., Bulligan G., A. CABrero, Ferrara L., StAHL H., 2010, Housing cycles in the major euro area countries, Documentos Ocasionales, no. 1001, Banco de Espana, http://dx.doi.org/ 10.2139 /ssrn.1582354

Bandt de O., Knetsch T., Peñalosa J., Zollino F., 2010, Housing Markets in Europe: A Macroeconomic Perspective, Springer Berlin Heidelberg

BEŁEJ M., 2012, Dynamika zmian cen nieruchomości w aspekcie teorii przejść nieciagłych (Dynamics of Changes in Property Prices in Aspects of Discountionous Chane Theory), Studia i Materiały Towarzystwa Naukowego Nieruchomości, vol. 20, nr 1, Olsztyn, pp.17-28

BEŁEj M., 2013, Catastrophe Theory in Explaining Price Dynamics on the Real Estate Market, Real Estate Management and Valuation, Volume 21, Issue 3, Pages 51-61, DOI 10.2478/remav-2013-0026

BeŁej M., Kulesza S., 2014, Similarities in Time-Series of Housing Prices on Local Markets in Poland, Real Estate Management and Valuation. Volume 22, Issue 3, Pages 45-53, ISSN (Online) 2300-5289, DOI: https:/ / doi.org/10.2478/remav-2014-0026, October 2014

BeŁej M., Kulesza S., 2015, The Dynamics Of Time Series Of Real Estate Prices, Real Estate Management and Valuation. Volume 23, Issue 4, Pages 35-43, ISSN (Online) 2300-5289, DOI: https:/ / doi.org/10.1515/remav-2015-0034, January 2016

BIS Residential Property Price database (online: http:/ / www.bis.org/statistics/pp.htm)

BRACKE P. (2011), How Long Do Housing Cycles Last? A Duration Analysis for 19 OECD Countries, IMF Working Paper WP/11/231, http:/ / dx.doi.org/10.5089/9781463921316.001.

CELlMER R., 2010, Analiza przestrzenna dynamiki zmian cen nieruchomości lokalowych z wykorzystaniem regresji ważonej geograficznie (Spatial Analysis Of Dynamics Of Changes Housing Prices With Use Of Geographically Weighted Regression), Acta Scientiarum Polonorum. Administratio Locorum vol. 9 (4), pp. 5-14 
CESA-BiAnCHI A., Cespedes L. F., Rebucci A., 2015, Global Liquidity, House Prices, and the Macroeconomy: Evidence from Advanced and Emerging Economies. Journal of Money, Credit and Banking, 47: 301-335. doi: $10.1111 /$ jmcb.12204

DitTMANN I., 2012a, Lokalne rynki mieszkaniowe w Polsce - podobieństwo pod względem zmian cen transakcyjnych oraz dostępności mieszkań (The Local Residential Markets in Poland - Similarity in Terms of Changes in Transaction Prices and Availability), "Studia i Materiały Towarzystwa Naukowego Nieruchomości", vol. 20, nr 1, pp. 71-89, Olsztyn

DitTMAnN I., 2012b, Prognozowanie cen na lokalnych rynkach nieruchomości mieszkaniowych na podstawie analogii przestrzenno - czasowych (Forecasting Prices on Residential Real Estate Local Markets Based on Area-Time Analogies), Ekonometria Econometrics nr 4 (38), Wydawnictwo Uniwersytetu Ekonomicznego we Wrocławiu, pp. 93-105.

DitTManN I., 2013, Podobieństwo zmian średnich cen transakcyjnych 1 m2 powierzchni mieszkań w wybranych miastach województwa ślaskiego (The Similarity of the Changes in Average Transaction Prices of $1 \mathrm{~m} 2$ of Housing in Selected Cities of Silesia), Studia Ekonomiczne - Zeszyty Naukowe Uniwersytetu Ekonomicznego w Katowicach, nr 124, Wyd. UE w Katowicach, pp. 165 - 182

DitTMAnN I., 2014, Gamma konwergencja cen na lokalnych rynkach mieszkaniowych w Polsce (Gamma Convergence Of Prices On Local Housing Markets In Poland), Studia Ekonomiczne. Zeszyty Naukowe Uniwersytetu Ekonomicznego w Katowicach, Uniwersytet Ekonomiczny w Katowicach, nr 181, 2014, pp. 195-207

ENGSTED T., PETERSEN T.Q., 2015, Predicting returns and rent growth in the housing market using the rentprice ratio: Evidence from the OECD countries, Journal of International Money and Finance, Volume 53, May 2015, Pages 257-275

GNAT S., 2016, Tests for the Presence of Price Convergence on Residential Property Market in Several Districts of Szczecin in 2006-2009, Folia Oeconomica Stetinensia. Volume 16, Issue 1, Pages 186-195, ISSN (Online) 1898-0198, DOI: https:/ / doi.org/10.1515/foli-2016-0011

HorsewoOd N., 2011, Demystifying Quantitative Methods in Comparative Housing Research: Dispelling the Myth of Black Magic, International Journal of Housing Policy, Vol. 11, Issue 4, pp. 375-393, DOI: 10.1080/14616718.2011.626601

IGAN D., LOUNGANI P., 2012, Global Housing Cycles, IMF Working Paper, WP/12/217, August

Kydland F. E, Rupert P., Šustek R., 2016, Housing Dynamics Over The Business Cycle, International Economic Review, Volume 57, Issue 4, Pages 1149-1177, DOI: 10.1111/iere.12193

KRUSZKA M.,TRojANEK R., 2014, The Synchronization of Price Cycles in the Local Housing Markets in Poland in 1996-2012. Świat Nieruchomości, 4(90), 5-11. DOI: 10.14659/worej.2014.90.01

MiLCHEVA S., ZHU B., 2016, Bank integration and co-movements across housing markets, Journal of Banking \& Finance, Volume 72, Supplement, November 2016, Pages S148-S171, DOI: 10.1016/j.jbankfin.2015.07.002

Miles W., 2015, Regional House Price Segmentation and Convergence in the US: A New Approach, January 2015, Volume 50, Issue 1, pp 113-128, DOI: 10.1007/s11146-013-9451-y

SCATIGNA M., SZEMERE R., TSATSARONIS K., 2014, Residential property price statistics across the globe, BIS Quarterly Review, September 2014 (dostęp online: http://www.bis.org/publ/qtrpdf/r_ qt1409h.htm)

SCHINDleR F., 2013, Predictability and Persistence of the Price Movements of the SEP/Case-Shiller House Price Indices, The Journal of Real Estate Finance and Economics, January 2013, Volume 46, Issue 1, pp 44-90, doi:10.1007/s11146-011-9316-1

STEINER K., 2013, Residential Property Prices in Central, Eastern and Southeastern European Countries: Stocktaking of Data and a View on New Developments in Data Availability, Focus on European Economic Integration, 3rd Quarter 2013, pp. 82-94

SZANDUŁA J., analogie_makro2.xls, (online: http:/ / szandulajacek.republika.pl)

TISSOT B., 2014, Monitoring house prices from a financial stability perspective - the BIS experience, November (online: http://www.bis.org/ifc/events/ISI_reg_stats_conf2014/session4_BISpaper.pdf)

Titman S., WANG K., YANG J., 2014, The Dynamics of Housing Prices, Journal of Real Estate Research, Volume 36, Number 3, pp. 283-317

TrojAneK R., 2012, An Analysis Of Changes In Dwelling Prices In The Biggest Cities Of Poland In 2008-2012 Conducted With The Application Of The Hedonic Method, Actual Problems of Economics, pp. 5-14 
TSATSARONIS K., ZHU H., 2004, What drives housing price dynamics: cross country evidence, BIS Quarterly Review, March, pp. 65-78.

Weise A.D., PHILIPS J.W., HochHeIM N., 2015, Cyclicity of Housing Markets Under the Specific Condition of the Existence of a Bubble in the Real Estate Market, Real Estate Management and Valuation, Vol. 23, No. 3, pp. 85-98, DOI: 10.1515/remav-2015-0028

WOLSKI R., 2016, Investment Risk in the Context of Price Changes on the Real Estate and Stock Markets, Real Estate Management and Valuation, Vol. 24, No. 1, pp. 41-50, DOI: https://doi.org/10.1515/remav-2016-0004

ZiMmeR, D.M., 2015, Time-Varying Correlation in Housing Prices, The Journal of Real Estate Finance and Economics, July 2015, Volume 51, Issue 1, pp. 86-100, doi:10.1007/s11146-014-9475-y

ŻELAZOWSKI K., 2011, Regionalne zróżnicowanie cen $i$ ich determinant na rynku mieszkaniowym w Polsce (Regional Differences in Prices and Their Determinants on Polish Housing Market), Studia i Materiały Towarzystwa Naukowego Nieruchomości, vol. 19 nr 3, Olsztyn, pp. 98-106.

ŻELAZOWSKI K., 2016, Fluktuacje cen na rynkach mieszkaniowych w kontekście cykli kredytowych (Price fluctuations in housing markets in the context of credit cycles), referat wygłoszony na Ogólnopolskiej Konferencji Naukowej „Finanse - Statystyka - Badania empiryczne” zorganizowanej przez Katedrę Finansów oraz Katedrę Prognoz i Analiz Gospodarczych na Uniwersytecie Ekonomicznym we Wrocławiu w dniu 26.10.2016 